\title{
A Fibrin-Based Tissue-Engineered Renal Proximal Tubule for Bioartificial Kidney Devices: Development, Characterization and In Vitro Transport Study
}

\author{
Chee Ping Ng, ${ }^{1,2}$ Yuhang Zhuang, ${ }^{1}$ Alex Wei Haw Lin,, ${ }^{1,3}$ and Jeremy Choon Meng Teo ${ }^{1,4}$ \\ ${ }^{1}$ Institute of Bioengineering and Nanotechnology, 31 Biopolis Way, The Nanos, Singapore 138609 \\ ${ }^{2}$ BioSystems and Micromechanics (BioSyM) IRG, Singapore-MIT Alliance for Research and Technology (SMART), \\ 1 CREATE Way, No. 10-01 CREATE Tower, Singapore 138602 \\ ${ }^{3}$ School of Engineering, Republic Polytechnic, 9 Woodlands Avenue 9, Singapore 738964 \\ ${ }^{4}$ Department of Biomedical Engineering, Khalifa University of Science Technology and Research, P.O. Box 127788, Abu Dhabi, UAE
}

Correspondence should be addressed to Chee Ping Ng; ng.chee.ping@smart.mit.edu

Received 31 August 2012; Accepted 24 October 2012

Academic Editor: Miguel Oliveira

Copyright (C) 2013 Chee Ping Ng et al. This is an open access article distributed under the Creative Commons Attribution License, which permits unrestricted use, distribution, and reproduction in any medium, provided the original work is properly cited.

\begin{abstract}
A bioartificial renal proximal tubule is successfully engineered as a first step towards a bioartificial kidney for improved renal substitution therapy. To engineer the tubule, a tunable hollow fiber membrane with an exterior skin layer that provides immunoprotection for the cells from extracapillary blood flow and a coarse inner surface that facilitates a hydrogel coating for cell attachment was embedded in a "lab-on-a-chip" model for the small-scale exploratory testing under flow conditions. Fibrin was coated onto the inner surface of the hollow fiber, and human renal proximal tubule epithelial cells were then seeded. Using this model, we successfully cultured a confluent monolayer, as ascertained by immunofluorescence staining for ZO-1 tight junctions and other proximal tubule markers, scanning electron microscopy, and FITC-inulin recovery studies. Furthermore, the inulin studies, combined with the creatinine and glucose transport profiles, suggested that the confluent monolayer exhibits functional transport capabilities. The novel approaches here may eventually improve current renal substitution technology for renal failure patients.
\end{abstract}

\section{Introduction}

Hemodialysis has been widely used to treat patients suffering from end-stage renal failure. With hemodialysis, millions of renal failure patients in the world have not only survived, but are also able to successfully perform certain levels of social activities and functions. However, hemodialysis is still inefficient as a renal replacement therapy. It only provides intermittent blood filtration and is unable to replicate the important absorptive, metabolic, endocrine, and immunological functions of the natural kidney. To overcome such limitations, Aebischer et al. first proposed the concept of a bioartificial kidney that incorporates living epithelial cells of the kidney proximal tubule for the selective transport of water and solutes across the cell-attached membranes $[1,2]$. Humes et al. have developed a bioartificial renal tubule assist device (RAD) using commercial hemodialysis hollow fiber membranes based on this concept [3-6] and conducted clinical trials on 10 to 40 patients with acute renal failure; encouraging results were reported $[7,8]$. Saito et al. also developed a bioartificial renal tubule device (BRTD) with a capacity of treating $10 \mathrm{~L}$ of ultrafiltrate per day. Using proximal tubular epithelial cells in conjunction with continuous hemofiltration, the BRTD offers a potential wearable, continuous renal replacement therapy for patients with chronic renal failure [9-14].

In the development of a bioartificial kidney device, it is critical to attain an appropriate hollow fiber membrane supporting a confluent monolayer of kidney cells to perform the renal tubule functions. This membrane must provide immunoprotection for the kidney cells on the inner surface from the attack of the antibodies in the blood flowing outside the hollow fibers. It should also serve as a barrier that prevents albumin and other large molecules in the blood from leaking 
into the filtrate through small gaps in the case of a nonconfluent cell layer. Previous researchers employed commercially available fibers, such as polysulfone hollow fibers from Minntech Diafilter [3] and Fresenius F40 [4, 7], polyimide and polysulfone hollow fibers from Minntech [12], Nipro [10] and Millipore [14], and ethylene vinylalcohol copolymer fibers from Kuraray [12]. The commercially available fibers may not be the optimal choice for reabsorption applications for the following reasons. Firstly, they are intended for hemofiltration, hemodialysis, or other filtration processes, whereby the feed (i.e., blood) usually flows inside the fibers. Accordingly, the skin layer is designed to be on the inner surface of the fibers, facing the liquid to be filtered [15], while the outer surface usually has pores as large as $1 \mu \mathrm{m}$ so as to lower filtration resistance. To perform reabsorption function, however, the filtrate should flow through the lumen and the blood should flow outside the fibers. In using the commercial filtration fibers for reabsorption, large molecules in the blood that are to be rejected (including blood cells, albumin, and antibodies) may be trapped in the large pores on the outer fiber surface, resulting in the loss of important serum substances. Problem may also arise with clogging of the membrane, with high resistance against transferring the reabsorbed substances back to blood. In addition, the reabsorption conditions (e.g., flow rate, pressure, and solute transport requirement) are quite different from those in dialysis. The optimal fiber diameter, pore size distribution, and mechanical strength for reabsorption may not be the same as those designed for filtration. Also, the filtrate flow is $~ 19 \%$ of the blood flow, and $99 \%$ of this filtrate should be reabsorbed back to the blood [16]. Thus, the total flow rate through the lumen of the reabsorption fibers would be much less than in the filtration fibers. This would affect the selection of fiber diameter and number of fibers to be employed for reabsorption. Furthermore, the inner surface of the hollow fiber membrane must support a confluent monolayer of renal epithelial cells. The inner surface of current commercial filtration fibers is very smooth (pores in the skin layer are $<7 \mathrm{~nm}$ to stop albumin transport); this would not favor the adhesion of the coating materials and cells. Thus, a relatively porous inner fiber surface would enhance coating adhesion for cell attachment. As a result, a tunable hollow fiber membrane with an exterior skin layer that provides immunoprotection for the cells from extracapillary blood flow and a coarse inner surface that facilitates a hydrogel coating for cell attachment was developed previously [17].

In addition, to improve cell attachment, proliferation, and differentiation on the hollow fiber membrane, coating materials such as pronectin-L [5], pronectin-F [10], laminin $[4,6,18]$, and bovine collagen type IV $[4,14]$ were applied in previous devices. However, such extracellular matrix materials are typically expensive and limited, particularly if human sources are needed, while animal sources, while abundant, face regulatory issues in part due to possible transmissions or contamination of undesirable agents such as endotoxins, viruses, or even Bovine Spongiform Encephalopathy (BSE)causing prions [19]. In addition, the cost and abundance may be an issue in the subsequent scale-up and large-scale employment of such devices. Furthermore if a small quantity (typically in $\mu \mathrm{g} / \mathrm{mL}$ ) is used for coating, this might lead to poor attachment of cells to the hollow fiber membrane. Indeed, cell detachment in the devices had been reported previously up to $40 \%$ of the membrane area under perfusion conditions $[3,11]$, leading to suboptimal performance of these devices. Here, we proposed and studied the viability of using fibrin as a cell attachment and differentiation matrix for renal proximal tubule cells. Fibrin is formed by the polymerization of fibrinogen in the presence of the enzyme thrombin [20-23]. It is a provisional matrix that is eventually replaced by the cells' own ECM. The material is inexpensive, approved by FDA as a tissue sealant for clinical applications, and can be autologous (directly isolated from the patients' blood plasma). Due to these advantages, fibrin is commonly used in tissue engineering applications such as the endothelialization of PTFE grafts $[24,25]$ for vascular engineering. However, its potential use for renal tubule epithelialization of hollow fibers has not been investigated.

Herein we described a novel ECM-hollow fiber membrane combination that could significantly reduce protein adsorption in the outer surface and enhance renal cell attachment on the inner surface. Human renal proximal tubule epithelial cells were successfully cultured as a confluent monolayer and exhibit transport functions. The resulting engineered tubule platform represents a useful and significant step in the development of a bioartificial kidney device.

\section{Materials and Methods}

2.1. Preparation of Hollow Fiber Membranes. Hollow fiber membranes were fabricated as previously described [17]. Briefly, polyethersulfone (PES, average molecular weight $(\mathrm{MW})=51 \mathrm{kDa}, \mathrm{BASF})$ and polyvinylpyrrolidone (PVP, average $\mathrm{MW}=25 \mathrm{kDa}$, Merck) were slowly added to $\mathrm{N}$ methyl-2-pyrrolidone (NMP) in a glass bottle at the desired loading (18 and $10 \mathrm{wt} \%$, resp., unless otherwise specified) and stirred until a homogeneous dope solution was obtained. The bore solution was a biocompatible oil, FC3283 (3 M), which is immiscible with neither water nor NMP, with a density of $1820 \mathrm{~kg} / \mathrm{m}^{3}$ and a viscosity of $1.4 \mathrm{cp}$. The dope and the bore solutions were pumped into an extruder at flow rates of 0.08 and $0.05 \mathrm{~mL} / \mathrm{min}$, respectively [26]. The exit of the extruder was immersed in a coagulation bath composed of $10 \mathrm{vol} \% \mathrm{NMP}$ in $\mathrm{H}_{2} \mathrm{O}$ to perform wet spinning at room temperature.

The resulting hollow fibers were guided by rollers and wound up by a collection wheel at a speed of $0.3 \mathrm{~m} / \mathrm{min}$. Next, the fibers were immersed in deionized (DI) water for at least $24 \mathrm{hr}$ to remove residual organic solvent. They were cut into segments of desired length and flushed with sufficient DI water to remove the FC3283 oil in the fiber lumen. One set of samples was freeze-dried and characterized by scanning electron microscopy (SEM). Another set of samples was immersed in a $35 \mathrm{wt} \%$ glycerol aqueous solution for 1 day before drying at $70^{\circ} \mathrm{C}$ for $24 \mathrm{hr}$. These fibers were rinsed extensively and autoclaved at $120^{\circ} \mathrm{C}$ for $30 \mathrm{~min}$ for hydrogel coating and cell culture. 
2.2. "Lab-on-a-Chip" Bioreactor System. The system is composed of a 1-inch hollow fiber membrane pretreated with $40 \%$ glycerol in PBS, a polydimethylsiloxane (PDMS) body and a glass substrate. The PDMS body is created with apical and basal chamber features using soft lithography by mixing Sylgard 184 base and curing agent (Dow Corning) in the weight/weight ratio of $10: 1$, followed by applying the elastomer mix into a Fullcure mold printed by a Polyjet rapid prototyping machine (Objet Geometries Ltd, Rehovot, Israel). The PDMS is cured for $4 \mathrm{hrs}$ in a $70^{\circ} \mathrm{C}$ oven, removed from the mold, and further cured overnight at $70^{\circ} \mathrm{C}$. Finally the hollow fiber, after being treated with $40 \%$ glycerol for at least 1 week, is dried and assembled into the PDMS body, which is later bonded to a glass microscope slide using silicone glue (Dow Corning).

\subsection{Preparation and Perfusion of Cell-Seeded Hollow Fiber} Membrane in Bioreactor System. Using a syringe pump (New Era Pump Systems Inc., Wantagh, NY), the chamber was perfused continuously with $70 \%$ ethanol for $6 \mathrm{hrs}$ and subsequently with deionized water for 3 days at a flow rate of 1$2 \mathrm{~mL} / \mathrm{min}$ to wet out the dried hollow fiber and remove the trapped glycerol.

To perform the fibrin coating, the hollow fiber membrane lumen was incubated with $5 \mathrm{mg} / \mathrm{mL}$ bovine fibrinogen (Sigma Aldrich, St. Louis, USA) in phosphate buffer saline (PBS) for $1 \mathrm{hr}$ at $37^{\circ} \mathrm{C}$, then subsequently overnight at $4^{\circ} \mathrm{C}$. Excess and unbound fibrinogen in the hollow fiber was removed by rinsing with sterile PBS before the introduction of $50 \mathrm{U} / \mathrm{mL}$ bovine thrombin (Sigma Aldrich) for 5-10 minutes to cross-link the adsorbed fibrinogen. Any unbounded thrombin and hydrogel were then flushed out of the fiber with thorough rinsing of sterile PBS.

Human primary renal proximal tubule epithelial cells (RPTEC; Lonza Walkersville Inc, Walkersville, Maryland, USA) were grown in tissue culture flasks (Iwaki, Tokyo, Japan) using Renal Epithelial Growth Medium (REGM; Lonza) up to $80 \%$ confluency before use. Only cells up to passage 4 were used for the experiments. The cells were dislodged from the culture flasks with EDTA/Versene. After rinsing at low speed centrifugation, the cells were suspended in the experimental medium consisting of Epithelial Cell Medium-phenol red free medium (EpiCM-prf; ScienCell Research Laboratories, Carlsbad, CA) supplemented with epithelial cell growth supplement (EpiCGS; ScienCell) and penicillin/streptomycin solution (ScienCell) at a concentration of $5 \times 10^{6}$ cells $/ \mathrm{mL}$ and introduced into the fiber lumen. The cells were incubated at $37^{\circ} \mathrm{C}, 5 \% \mathrm{CO}_{2}$ for 90 minutes to allow for cell adhesion. The minibioreactor was rotated $90^{\circ}$ and another seeding of cells was performed. This was repeated 2 more times. In total, 4 seedings of cells were done for complete seeding of the tubular hollow fiber in the minibioreactor. The bioreactor was then perfused using a syringe pump (New Era Pump Systems Inc.) with a single pass of the experimental medium through the extracapillary space of the hollow fiber membrane for 2 days at $200 \mu \mathrm{L} / \mathrm{hr}$ before switching to intraluminal perfusion at $200 \mu \mathrm{L} / \mathrm{hr}$ under a humidified atmosphere of $5 \% \mathrm{CO}_{2}$ at $37^{\circ} \mathrm{C}$ (see Figure 1).
The bioartificial renal proximal tubules were cultured and conditioned under flow for at least 7 days before immunofluorescence staining and electron microscopy and 10-14 days before the inulin recovery transport studies to assess the functionality of confluent cell monolayer in bioreactor.

2.4. Immunofluorescence Staining. The primary rabbit polyclonal (gamma-glutamyl transpeptidase $(\gamma$-GTP), Megalin, Aquaporin-1 (AQP-1)) and mouse monoclonal antibodies (CD13, $\mathrm{Na}^{+} \mathrm{K}^{+}$adenosine triphosphatase $\left(\mathrm{Na}^{+} \mathrm{K}^{+}\right.$ATPase) were purchased from Santa Cruz Biotechnology Inc (Santa Cruz, California, USA). Rabbit ZO-1 antibody, Alexa Fluor 488 conjugated phalloidin and MitoTracker, secondary antibodies such as Alexa Fluor 488-conjugated goat-antirabbit, and Tetramethyl rhodamine isothiocyanate (TRITC)conjugated goat-anti-mouse IgG were from Invitrogen (Carlsbad, California, USA). Cy3 conjugated alpha-smooth muscle actin antibody was purchased from Sigma Aldrich while SGLT2 was from Abcam (Cambridge, UK). To perform immunofluorescence characterization, the cell-seeded fibrincoated fibers were preserved in $4 \%$ paraformaldehyde in PBS for 15 minutes and rinsed thoroughly with PBS. Only the section of fibers exposed to the extracapillary space was carefully removed from the bioreactor using a scalpel and cut into small sections for further processing. For surface antigens such as CD13, no permeabilization was performed prior to blocking with PBS with 1\% Bovine Serum Albumin (BSA; Sigma Aldrich) for 30 minutes to inhibit unspecific binding. Otherwise, permeabilization of the cells in the hollow fiber sections was performed with $0.25 \%$ Triton in PBS followed by blocking with PBS with $0.2 \%$ Tween (PBST) supplemented with $1 \%$ BSA. This is followed by overnight incubation of the primary antibodies at $4^{\circ} \mathrm{C}$. Secondary antibodies were applied to the sections for $1 \mathrm{hr}$ at room temperature before they were costained for the cell nuclei using Hoechst 33342 (Invitrogen).

2.5. Electron Microscopy. For electron microscopy, the cellcoated hollow fibers were first fixed in $2 \%$ glutaraldehyde in culture medium and then dried in ethanol solution at increasing volume percent concentrations $\left(35 \mathrm{vol} \%\right.$ in $\mathrm{H}_{2} \mathrm{O}$, 50 vol\% in $\mathrm{H}_{2} \mathrm{O}, 70 \mathrm{vol} \%$ in $\mathrm{H}_{2} \mathrm{O}, 80 \mathrm{vol} \%$ in $\mathrm{H}_{2} \mathrm{O}, 95 \mathrm{vol} \%$ in $\mathrm{H}_{2} \mathrm{O}, 100$ vol\% and 100 vol\%) for $60 \mathrm{~min}$ each. The samples were next dried in an Autosamdri 825 critical point dryer. They were cut at an angle of $\sim 45^{\circ}$ from the fiber axis, such that part of the inner surface was exposed for SEM characterization.

2.6. Transport Characterization. Transport characterization was assessed by inulin recovery studies under flow and by measuring the urea, creatinine, and glucose profiles in a static configuration. The inulin recovery studies are based on the methodology established by MacKay and coworkers [6] with slight modifications. To assess the confluent cell monolayer along the inner surface of the hollow fiber and quantify the inulin recoveries, FITC-inulin (Sigma Aldrich) at a concentration of $40 \mathrm{mg} / \mathrm{dL}$ was perfused into the minibioreactor using a syringe pump set at $2.5 \mathrm{~mL} / \mathrm{hr}$. Samples were collected from the lumenal exit port to determine the concentration 

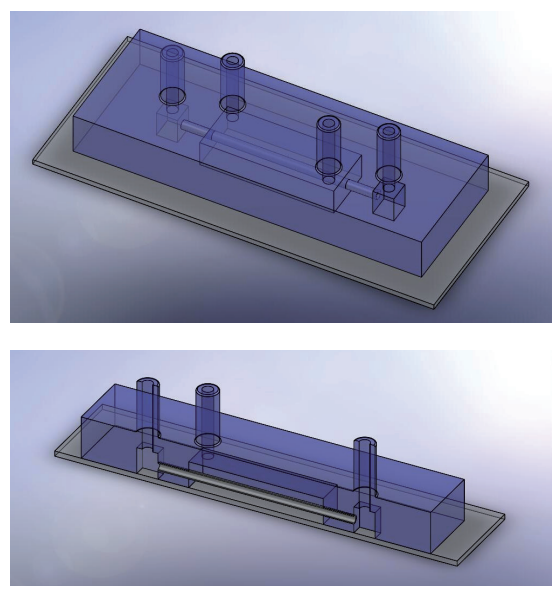

(a)

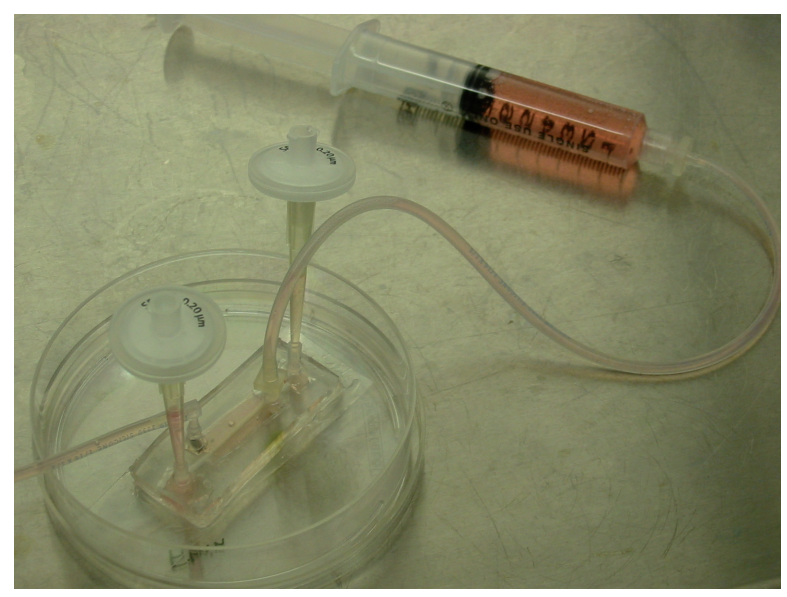

(b)

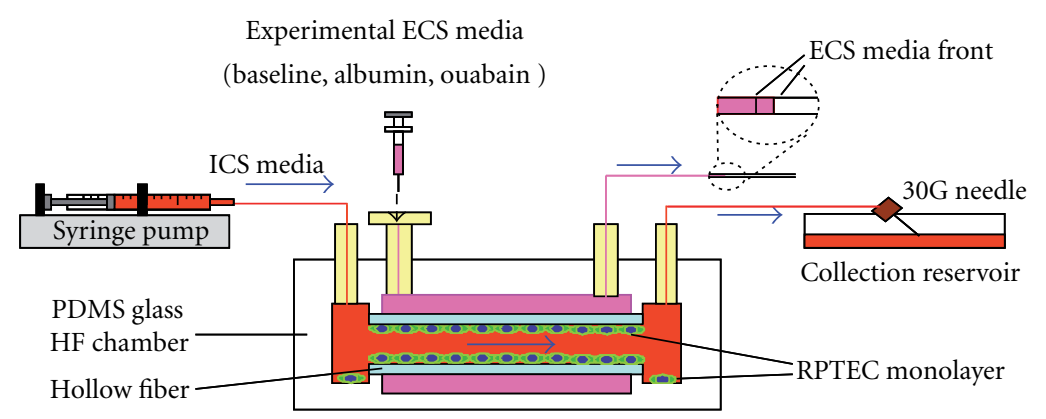

(c)

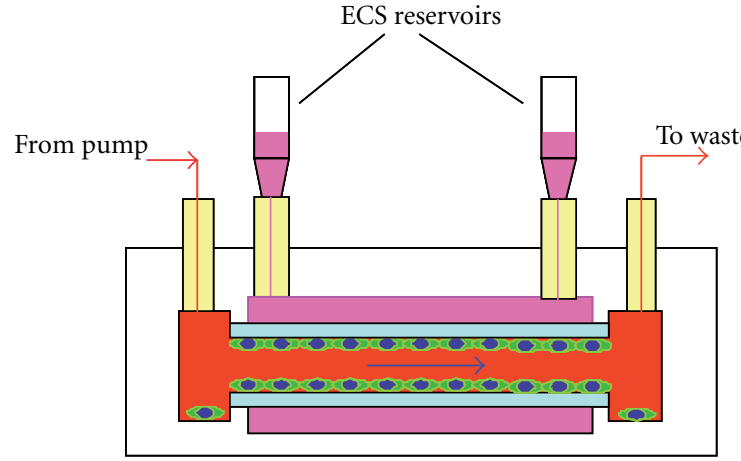

(d)

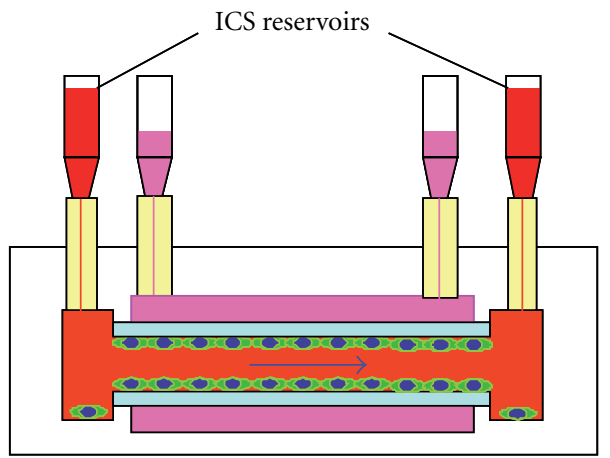

(e)

FIGURE 1: (a) Schematic and (b) image of the "lab-on-a-chip" hollow-fiber bioreactor. Set-up for (c) inulin recovery perfusion studies, (d) normal perfusion culture, and (e) static configuration for urea, creatinine, and glucose transport studies.

for comparison to the infused concentration. A $0.03^{\prime \prime}$ ID Silastic tubing (Dow Corning) was connected to one of the ECS ports closer to the lumenal exit port with the other port capped. The reabsorption rate or the ECS outflow rate is determined by measuring the distance of the ECS effluent movement in the tubing at $10 \mathrm{~min}$ intervals for a total of $30 \mathrm{~min}$. Three experimental conditions were studied; the first was a baseline condition with the experimental medium in the ECS; the second was the experimental medium with albumin $(4.0 \mathrm{~g} / \mathrm{dL})$ to the ECS to simulate transport with an oncotic pressure gradient; the final condition was experimental medium with albumin and ouabain $(5 \mathrm{mmol} / \mathrm{L})$, a specific inhibitor of $\mathrm{Na}^{+} \mathrm{K}^{+}$ATPase. These solutions were injected into the ECS through the PDMS body at the capped port and allowed to equilibrate for 30 minutes before the start of measurement.

To elucidate the urea, creatinine, and glucose profiles of the system, media reservoirs made from $200 \mu \mathrm{L}$ yellow pipet tip were attached to the luminal and ECS ports. A pressure difference of $2 \mathrm{~cm} \mathrm{H}_{2} \mathrm{O}$ was imposed across the cell-hollow fiber, and samples $(5-30 \mu \mathrm{L}$ with same amount replaced) were collected each day for a total of 3 days and assayed using QuantiChrom kits (BioAssay Systems, Hayward, CA). For the glucose transport studies, DMEM:F12 medium (PAA Laboratories $\mathrm{GmbH}$, Pasching, Austria) supplemented with 10\% serum (Hyclone; Thermo Fisher Scientific, Waltham, 
TABLE 1: Transport characteristics of single fiber proximal renal tubules.

\begin{tabular}{|c|c|c|c|c|c|}
\hline & $\begin{array}{c}\text { Absolute } \\
\text { readsorption } \\
\text { rate }(\mu \mathrm{L} / \mathrm{hr})\end{array}$ & $\begin{array}{l}\text { Fractional } \\
\text { readsorption } \\
\text { rate }(\mu \mathrm{L} / \mathrm{hr})\end{array}$ & $\begin{array}{l}\text { FITC-inulin } \\
\text { recovery } \\
(\%)\end{array}$ & $\begin{array}{l}\text { FITC-inulin } \\
\text { concentration } \\
\text { ratio }\left(C_{\text {out }} / C_{\text {in }}\right)\end{array}$ & $\begin{array}{c}\text { Flux } \\
(\mathrm{LMH})\end{array}$ \\
\hline $\begin{array}{l}\text { Cell free } \\
\text { (baseline) }\end{array}$ & $200.1 \pm 59.1$ & $9.0 \pm 2.3$ & $88.6 \pm 4.7$ & $0.97 \pm 0.05$ & $30.3 \pm 9.0$ \\
\hline Baseline & $12.2 \pm 13.7$ & $0.5 \pm 0.6$ & $104.0 \pm 6.6$ & $1.05 \pm 0.06$ & $1.8 \pm 2.1$ \\
\hline Albumin & $25.9 \pm 18.5$ & $1.7 \pm 1.5$ & $104.0 \pm 9.8$ & $1.07 \pm 0.11$ & $3.9 \pm 2.8$ \\
\hline Ouabain & $18.7 \pm 16.7$ & $0.7 \pm 0.7$ & $101.9 \pm 6.5$ & $1.03 \pm 0.07$ & $2.8 \pm 2.5$ \\
\hline
\end{tabular}

Results are biologically replicated and expressed as mean \pm standard deviation; $n=4$.

MA, USA) was used since serum was required for detection of glucose metabolism [27].

\section{Results and Discussion}

The fibers used in the studies were developed for reabsorption and were previously characterized in terms of fiber structure, strength, solute rejection, and protein adsorption [17]. In brief, these readsorption hollow fibers are of outer diameter $(780 \mu \mathrm{m})$, inner diameter $(490 \mu \mathrm{m})$, and fiber thickness $(145 \mu \mathrm{m})$ and have a dope composition of $18 \% \mathrm{PES} / 10 \% \mathrm{PVP} / 72 \% \mathrm{NMP}$. The rough inner surface of the reabsorption fiber is a three-dimensional porous network of large pores of $\sim 0.5 \mu \mathrm{m}$, while the smooth outer skin surface consisted of dense nanometer-sized pores of <10 nm [17]. These features would prevent undesired substances from entering the fiber from the skin layer at the outer surface, while enhancing the attachment of ECM and cells in the porous inner surface. In contrast, commercial hemofiltration hollow fibers have smooth skin layers on the inner surface with a rough outer surface; the roughness may adversely affect the blood flow due to the trapping and clogging of large molecules from the blood (including blood cells, albumin, and antibodies) into the large pores of the membrane. The inner surface of current commercial filtration fibers is very smooth; this would be unfavorable for the adhesion of the coated materials. Thus, the hollow fiber membranes developed and employed here are more suitable to those for hemofiltration.

\subsection{Confluent Tubular Cell Monolayer Formation with Fibrin-} Coated Hollow Fibers. Human proximal tubule epithelial cells (RPTEC) were seeded and cultured under flow in the luminal space of the single hollow fiber coated with $5 \mathrm{mg} / \mathrm{mL}$ fibrinogen and $50 \mathrm{U} / \mathrm{mL}$ thrombin in a "lab-ona-chip" minibioreactor (Figure 1(d)). Without the fibrin coating, it was observed that very few cells attached to the inner surface of the hollow fiber membrane (see supplementary Figure 1 (available online at doi:10.1155/2013/319476)). The cross-section and transverse view of a representative immunostained section (Figures 2(a) and 2(b), resp., with close-ups in Figures 2(c) and 2(d)) showed the successful formation of a confluent cell monolayer along the luminal surface of the fibrin-based hollow fiber. Furthermore, the RPTEC monolayer displayed an intact mesh-wire-like
ZO1 morphology with no alpha-smooth muscle actin fiber expression (Figure 2(d)). The confluent cell monolayer in fibrin-coated hollow fiber also expressed proximal tubule markers (CD13, megalin, $\gamma$-GTP, numerous mitochondria) and ion transporters (AQP-1, $\mathrm{Na}^{+} \mathrm{K}^{+}$ATPase, SGLT2) (Suppl. Figure S2) with cell polarization (Suppl. Figure 2(d) insets). F-actin was reorganized to thick bundles at the cell periphery as previously observed by others under flow conditions [2830].

Electron microscopy images of the inner surface of the PES reabsorption fiber also revealed a confluent RPTEC monolayer (Figure 2(d)). Except for artifacts (e.g., voids left by cell detachment in the sample preparation process), the cells covered the entire inner surface of the hollow fiber and demonstrated the correct polarization with many microvilli facing the lumen (Figure 2(f) and inset). The presence of microvilli could significantly increase the surface area of cells; this would be important for reabsorbing the useful substances from the filtrate (in the lumen) back to the blood (flowing outside the hollow fiber). No microvilli were observed on the cells from the side in contact with the fiber, confirming the desired cell polarization for reabsorption application.

3.2. Transport Characteristics of Cell-Seeded Hollow Fibers. Inulin recovery studies were performed to elucidate the transport characteristics of the reepithelialized fibrin-coated hollow fiber (Table 1). The cell-free fibrin-coated hollow fiber (reabsorption rate: $200.1 \pm 59.1 \mu \mathrm{L} / \mathrm{hr}$ ) was significantly leakier than the cell-laden hollow fiber (baseline: $12.2 \pm$ $13.7 \mu \mathrm{L} / \mathrm{hr}$ ). The inulin recovery for the cell free was also significantly lower (averaged $88.6 \pm 4.9 \%$ ) when compared to the cell-seeded hollow fibers (>95\%). All the cell-loaded conditions registered ratios greater than 1 , implying that the inulin in the feed solution is being concentrated as water is being selectively reabsorbed. The average for inulin concentration ratio of the cell-free hollow fiber was 0.97 . This usually high concentration ratio for the cell-free hollow fiber may be due to the large reabsorption rate and small ECS chamber volume $(\sim 150 \mu \mathrm{L})$; fluid in the ECS was quickly replaced and equilibrated with the feed. The results suggested that inulin recovery tests may be more practical and useful to ascertain the confluent integrity of the cell monolayer along the hollow fiber than sample immunostaining for ZO-1 tight junction proteins [31] as it is not possible to reuse the fixed and stained cell-loaded hollow fiber afterwards. 

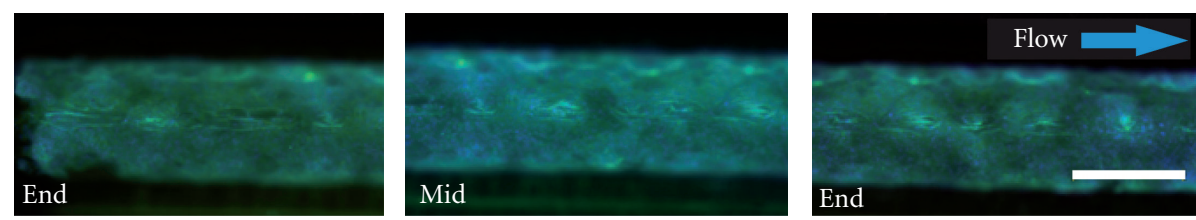

(a)

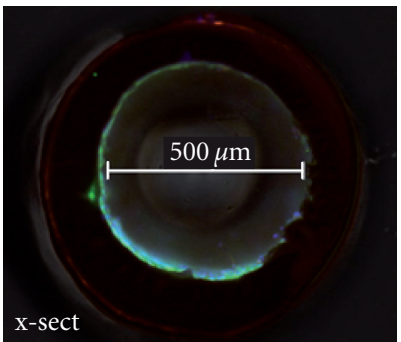

(b)

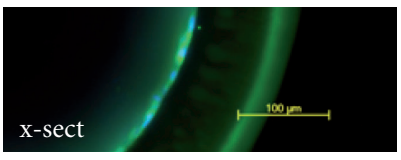

(c)

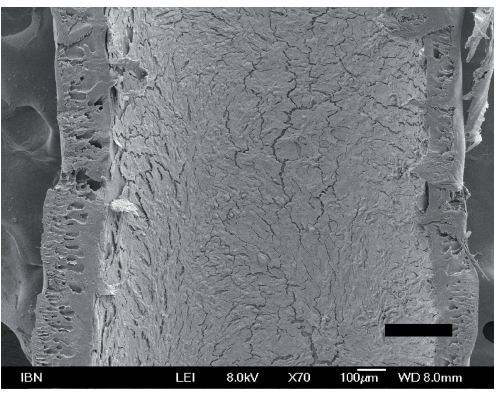

(e)

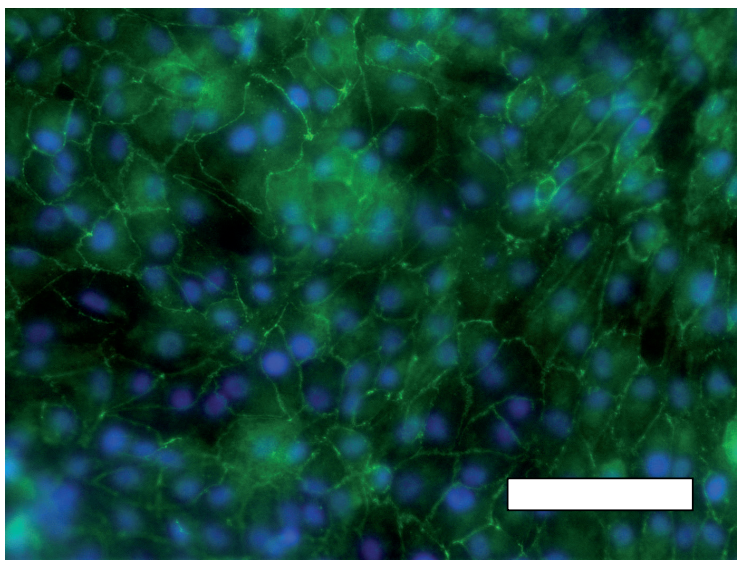

(d)

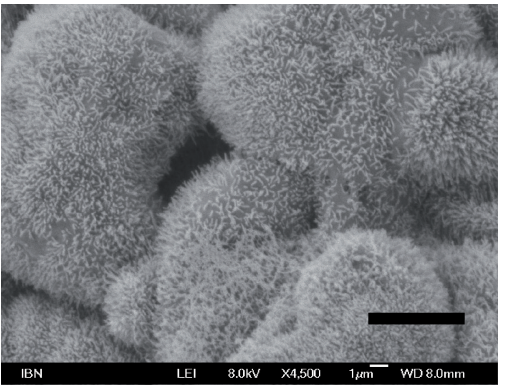

(f)

FIGURE 2: Functionality of cell-hollow fiber as assessed by immunofluorescence and electron microscopy. Epifluorescent images of consecutive sections of the immunostained cell-seeded hollow fiber for tight junction ZO-1 (green), $\alpha$-SMA, and Hoechst 33342 nuclei stain: (a) transverse and (b) cross-sectional view of a representative hollow fiber section (scale bar $=500 \mu \mathrm{m}$ ). Close-ups of the (c) cross-sectional view and (d) transverse view (confocal image projection) revealing a cell monolayer in the hollow fiber (scale bar $=100 \mu \mathrm{m}$ ). (e) Scanning microscope image of the cell-laden hollow fiber (black scale bar $=200 \mu \mathrm{m}$ ) with the (f) close-up showing microvilli expression on the cell surface (black scale bar $=5 \mu \mathrm{m})$. Some regions of the images are out of focus due to the curvature of the hollow fiber.

To test whether the renal tubule reabsorption observed with the baseline was driven by osmotic and oncotic pressures as is in vivo, we looked at the effects of albumin and ouabain addition in the ECS on various key transport indicators, particularly the reabsorption rate. The addition of albumin increased the absolute reabsorption rate to $25.9 \pm$ $18.5 \mu \mathrm{L} / \mathrm{hr}$ (Table 1). Adding ouabain, a specific inhibitor of $\mathrm{Na}^{+} \mathrm{K}^{+}$ATPase, attenuated this effect and reduced the reabsorption rate to $18.7 \pm 16.7 \mu \mathrm{L} / \mathrm{hr}$, closer to baseline levels. The large variation in reabsorption rates observed is due to the biological replications of different RPTECladen hollow fibers used but the trend between the baseline, albumin, and albumin + ouabain is the same for each hollow fiber (Figure 3). These results, particularly the ouabain data, suggested that the fluid reabsorption observed with the renal epithelialized fibrin coated hollow fibers is mediated by active transport.

Furthermore, we also looked at the urea, creatinine, and glucose transport profiles across the cell-lined hollow fiber under static conditions up to 3 days (Figure 4). The increase in urea concentration at the basal (ECS) side suggested reabsorption of urea across the intact epithelium-hollow fiber; this may be normal as approximately 50\% of urea in the ultrafiltrate is reabsorbed back into the peritubular capillaries. However similar to creatinine not absorbed across the proximal tubule epithelium in vivo, there was virtually no creatinine leakage across the cell-hollow fiber as shown by the unchanged concentration for the ECS (basal) sample; this also indicated the confluency of the cell monolayer lining the hollow fiber. Glucose transport was detected for at least a day 


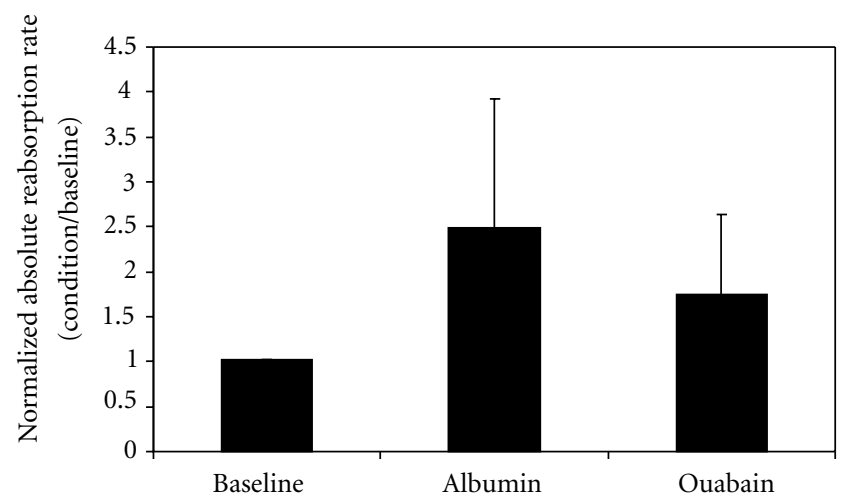

FIgURE 3: Normalized reabsorption rate (to baseline) of single fiber bioartificial proximal renal tubules subjected to baseline, albumin, and albumin + ouabain conditions. The biologically replicated data is represented as mean \pm standard deviation; $n=4$.

as determined by an increase in glucose concentration in the ECS side from $306 \pm 24 \mathrm{mg} / \mathrm{dL}$ to $317 \pm 20 \mathrm{mg} / \mathrm{dL}$. The volume of the apical (ICS) side for the urea, creatinine, and glucose did not change much since we are using a larger volume $(\sim 750 \mu \mathrm{L})$ compared to the basal side $(\sim 250 \mu \mathrm{L})$; therefore the concentration on the apical (ICS) side was less sensitive to change. Overall, the transport studies in combination with our immunofluorescence characterization suggest that fibrin offers a suitable cell attachment platform for RPTEC monolayer formation under perfusion conditions. Thus, a functional renal proximal tubule is successfully engineered here.

3.3. Discussion. The results here represent the initial important steps in the development of a bioartificial renal assist device to supplement current renal substitution therapy by adding reabsorptive, metabolic, and endocrine functions of the kidney. Although this concept is not new and goes back for more than 20 years, our research here offers new approaches to the field with hollow fiber membranes that are customized for engineering renal tubules and a previously unused extracellular matrix material for renal epithelialization in these membranes.

Previously, expensive and limited extracellular matrix materials are typically used for coating the inner surface of the hollow fiber to attach the renal cells. As a result, a small quantity $(\mu \mathrm{g} / \mathrm{mL})$ is used, and this might not sufficiently support cell attachment to the hollow fiber membrane. Since fibrin is inexpensive, more coating material may be used $(\mathrm{mg} / \mathrm{mL})$. Here, we demonstrated that fibrin is an excellent cell attachment matrix in our hollow fiber membranes as it promoted the differentiation of renal proximal tubule cells into confluent cell monolayer along the inner surface. This may be due to the fact that fibrin is a provisional wound healing matrix that is replaced by the ECM secreted by the cells. Thus, we allowed the cells to develop their own ECM which in turn resulted in excellent epithelialization. The promising results here demonstrated its feasibility and that a renal proximal tubule can be successfully created using fibrinbased technology.
In the process, we have also established a useful "lab-ona-chip" bioreactor system with methodologies for small-scale testing and optimization of RPTEC and hollow fiber membrane performance under flow conditions. This minibioreactor is useful for low-cost exploratory research since it might be expensive to set up a large-scale cartridge to test the viability of a hollow fiber membrane or coating material. In addition, the minibioreactor may have applications in pharmacology and toxicology as our model mimics in vivo closer in terms of flow and 3D tubular configuration. Also, it would be important to determine the long-term viability of the confluent monolayer along the hollow fiber using blood ultrafiltrate instead of culture medium. Preliminary studies with the experimental medium at a flow rate of $200 \mu \mathrm{L} / \mathrm{hr}$ showed cell confluency up to 22 days (data not shown). It would also be interesting to elucidate the effects of fibrin concentration, the magnitude of flow rate and medium content on the proliferation and maintenance of the cell monolayer grown on the fibrin coating in the hollow fiber.

A subsequent step of the project would be to develop a scale-up version of the engineered renal tubule. Preliminary calculations show that our technology has the potential to overcome disadvantages associated with commercial products mentioned previously. Based on the flux determined for the albumin condition (Table 1), a scale-up device with $1 \mathrm{~m}^{2}$ surface area can reabsorb approximately $3.9 \mathrm{~L}$ of fluid per hour. For continuous hemofiltration of 10 liters per day [10] where $99 \%$ of the blood ultrafiltrate is reabsorbed back, a $0.1 \mathrm{~m}^{2}$ surface area is required; this is an achievable surface area for an extracorporeal wearable device which we are interested to develop. Furthermore, one disadvantage of using commercial hollow fiber cartridges is that the specifications of these hemofiltration cartridges such as the fiber lengths and the number of fibers are fixed. For example, Humes et al. used large cartridges of fiber length $17 \mathrm{~cm}$ [5] while Ozgen et al. used cartridges of fiber length $17 \mathrm{~cm}$ and $34 \mathrm{~cm}$ [10]. These lengths might be ideal for high blood flow rates where hemofiltration is typically performed at the range of $300 \mathrm{~mL} / \mathrm{min}$. However, for slower flow in the range of $20 \mathrm{~mL} / \mathrm{min}$ as suggested by MacKay and coworkers [6] and used by Ozgen et al. [10], it is likely that the pressure drop across the long hollow fiber leads to unfavorable pressure gradient across the cell-hollow fiber layer towards the exit end, and consequently the performance of the device is suboptimal. Furthermore, the typical length of proximal tubules in vivo is about $1.4 \mathrm{~cm}$ where $67 \%$ of fluid is reabsorbed back in the nephron of the kidney. The effect of the fiber length on the reabsorption rates is an interesting subject that warrants further investigation. In addition, it may be better to "tailor-made" the renal tubule assist device according to each individual patient's physiological characteristics such as blood pressure. With our hollow fibers, we can customize not only the fiber diameter and fiber length but also the number of hollow fibers to optimize the performance of the engineered bioartificial renal tubules and meet each individual patient's specifications.

The results here suggest successful engineering of a functional bioartifical renal proximal tubule using our approach. 


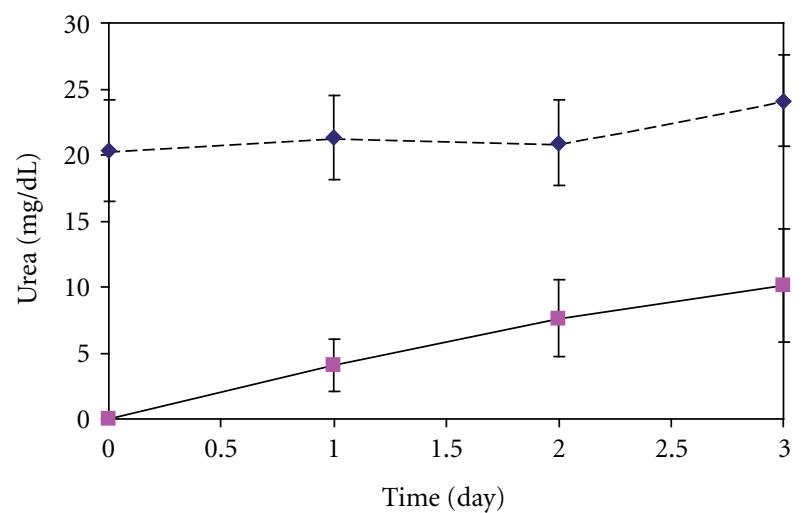

(a)

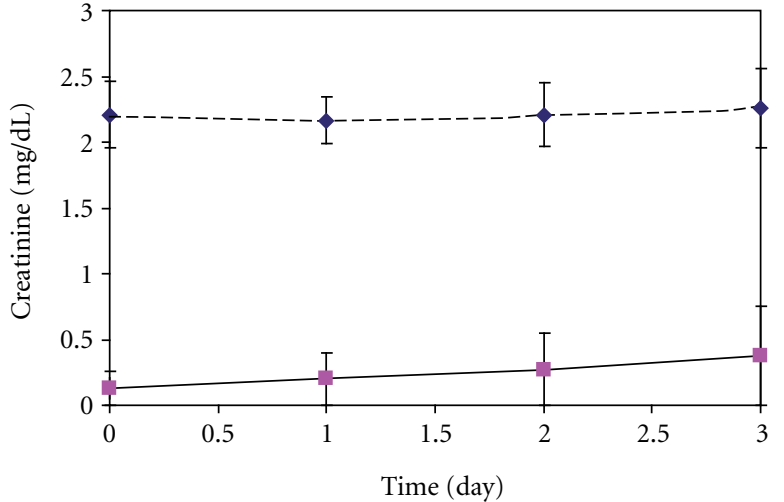

(b)

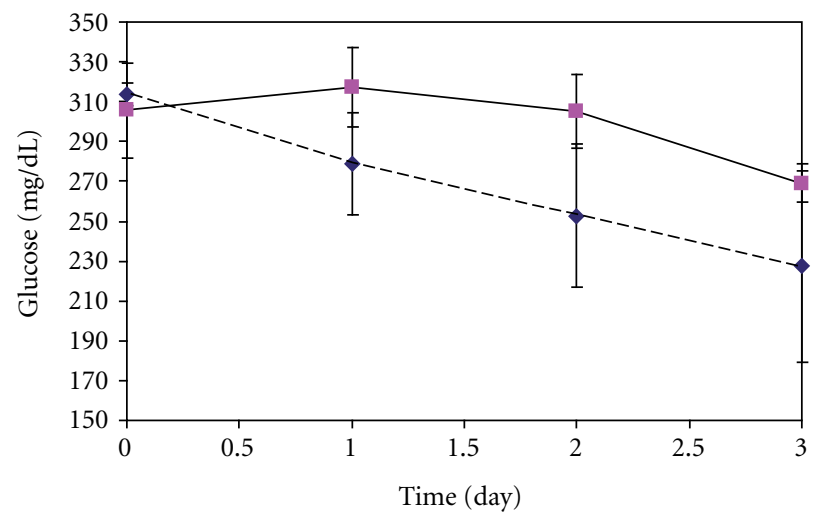

(c)

Figure 4: (a) Urea, (b) creatinine, and (c) glucose concentration in the luminal (dashed line) and ECS (solid line) compartment of the cellloaded hollow fiber under static conditions. The biologically replicated data is represented as mean $\pm \operatorname{standard}$ deviation; $n=3$.

However, the technology presented here is still in its infancy. Optimization such as membrane readsorption and bioactivity characteristics, hydrogel coating concentration, and the long-term viability of the cells is also needed for improved bioreactor performance. For example, membranes can be further developed to facilitate better adhesion of coating for improved cell monolayer proliferation, formation, and differentiation in the system $[32,33]$. In vivo evaluation is also required to assess the full functionality of the renal cells in the fibrin-based system. Nevertheless, many challenges remain, such as the limited and inexpensive source for healthy functional primary proximal tubule cells. For a full-scale device, up to $10^{9}$ functional cells per device may be required. While fresh primary human kidney cells have been used in such devices [18], it would be a challenge to procure such cells for mass production to meet the demand for mass production since healthy organs are prioritized for transplant. Some of the cell lines used previously in large-scale bioreactors are epithelial progenitor cells [5] and immortalized cells [10]. However, they typically do not exhibit the full functionalities found in primary cell lines although Wieser et al. have recently reported the successful immortalization of human proximal tubule epithelial cells without transformation or loss in functionalities using forced expression of hTERT [34]. Another approach that researchers have taken is to transfect a stable and poorly functional kidney cell line such as MDCK and LLC-PK1 cells to improve their transport capabilities $[9,35]$. For example, Saito et al. have transfected and studied AQP1-transfected LLC-PK1, reporting improved water transport capabilities of these cells on membranes [9]. Thus, it is then useful to multitransfect these stable or immortalized kidney cell lines with the various ion transporters such as SGLT2 and GLUT2 to improve cell performance. In addition, the hollow fiber membrane should be further developed to provide higher flux capabilities. Our cell-free fibrin-coated hollow fiber membranes have a calculated water permeability of $8.4 \times 10^{-4} \mathrm{~cm} / \mathrm{s}$. However, this is still significantly lower than that in mammalian proximal tubules $(0.07-0.7 \mathrm{~cm} / \mathrm{s})$ [5]. The water permeability of an ideal membrane for renal tubules should be equal to or higher than that value so that it is not a limiting factor for fluid reabsorption by these devices.

\section{Conclusions}

We have developed a hollow fiber membrane and investigated a cell adhesion coating for renal tubule engineering in a bioartificial kidney device. Unlike the conventional hollow fiber membranes employed in dialysis, our reabsorption hollow fiber membrane is designed with a skin layer at the outer 
fiber surface, which can reject undesired substances from the blood flow and minimize protein uptake on the fiber. Its inner surface is featured with pores of up to $0.3 \mu \mathrm{m}$ in size, facilitating the attachment of extracellular matrix coating on the membrane. Together with a fibrin coating, a confluent monolayer of human kidney renal proximal tubule epithelial cells is successfully formed on the fiber inner surface under flow conditions in a "lab-on-a-chip" bioreactor system. These cells demonstrate the correct polarization for reabsorbing the desired solutes from glomerular filtrate back to the blood with transport functionalities. The use of reabsorption hollow fiber membrane and fibrin coating for better support of renal cells provide very advantageous features for the development of an improved bioartificial kidney device.

\section{Conflict of Interests}

The authors declare that they do not have any direct financial relation with the commercial identities mentioned in the paper that might lead to a conflict of interests, such as financial gain.

\section{Acknowledgments}

The authors are grateful to Professor Jackie Y Ying and Dr. Daniele Zink for their helpful comments and Drs. Rensheng Deng and Min Hu for their technical assistance in the fabrication and electron microscopy of the hollow fiber membranes and in the preparation of the paper. This work was funded by the Institute of Bioengineering and Nanotechnology (IBN) and the Biomedical Research Council, Agency for Science, Technology and Research, Singapore (BMRC, A*STAR). Y. Zhuang was supported through the IBN Young Research Program (YRP).

\section{References}

[1] P. Aebischer, T. K. Ip, G. Panol, and P. M. Galletti, "The bioartificial kidney: progress towards an ultrafiltration device with renal epithelial cells processing," Life Support Systems, vol. 5, no. 2, pp. 159-168, 1987.

[2] T. K. Ip and P. Aebischer, "Renal epithelial-cell-controlled solute transport across permeable membranes as the foundation for a bioartificial kidney," Artificial Organs, vol. 13, no. 1, pp. 58-65, 1989.

[3] H. D. Humes, D. A. Buffington, S. M. MacKay, A. J. Funke, and W. F. Weitzel, "Replacement of renal function in uremic animals with a tissue- engineered kidney," Nature Biotechnology, vol. 17, no. 5, pp. 451-455, 1999.

[4] H. D. Humes, W. H. Fissell, W. F. Weitzel et al., "Metabolic replacement of kidney function in uremic animals with a bioartificial kidney containing human cells," American Journal of Kidney Diseases, vol. 39, no. 5, pp. 1078-1087, 2002.

[5] H. D. Humes, S. M. MacKay, A. J. Funke, and D. A. Buffington, "Tissue engineering of a bioartificial renal tubule assist device: in vitro transport and metabolic characteristics," Kidney International, vol. 55, no. 6, pp. 2502-2514, 1999.

[6] S. M. Mackay, A. J. Funke, D. A. Buffington, and H. David Humes, "Tissue engineering of a bioartificial renal tubule," ASAIO Journal, vol. 44, no. 3, pp. 179-183, 1998.
[7] H. D. Humes, W. F. Weitzel, R. H. Bartlett et al., "Initial clinical results of the bioartificial kidney containing human cells in ICU patients with acute renal failure," Kidney International, vol. 66, no. 4, pp. 1578-1588, 2004.

[8] J. Tumlin, R. Wali, W. Williams et al., "Efficacy and safety of renal tubule cell therapy for acute renal failure," Journal of the American Society of Nephrology, vol. 19, no. 5, pp. 1034-1040, 2008.

[9] Y. Fujita, M. Terashima, T. Kakuta et al., “Transcellular water transport and stability of expression in aquaporin 1-transfected LLC-PK1 cells in the development of a portable bioartificial renal tubule device," Tissue Engineering, vol. 10, no. 5-6, pp. 711-722, 2004.

[10] N. Ozgen, M. Terashima, T. Aung et al., "Evaluation of longterm transport ability of a bioartificial renal tubule device using LLC-PK1 cells," Nephrology Dialysis Transplantation, vol. 19, no. 9, pp. 2198-2207, 2004.

[11] A. Saito, "Research into the development of a wearable bioartificial kidney with a continuous hemofilter and a bioartificial tubule device using tubular epithelial cells," Artificial Organs, vol. 28, no. 1, pp. 58-63, 2004.

[12] A. Saito, T. Aung, K. Sekiguchi et al., "Present status and perspectives of bioartificial kidneys," Journal of Artificial Organs, vol. 9, no. 3, pp. 130-135, 2006.

[13] A. Saito, H. Suzuki, K. Bomsztyk, and S. Ahmad, "Regeneration of peritoneal effluent by Madin-Darby canine kidney cells-lined hollow fibers," Materials Science and Engineering C, vol. 6, no. 4, pp. 221-226, 1998.

[14] Y. Sato, M. Terashima, N. Kagiwada et al., "Evaluation of proliferation and functional differentiation of LLC-PK 1 cells on porous polymer membranes for the development of a bioartificial renal tubule device," Tissue Engineering, vol. 11, no. 9-10, pp. 1506-1515, 2005.

[15] R. W. Baker, Membrane Technology and Applications, John Wiley \& Sons, New York, NY, USA, 2nd edition, 2004.

[16] R. R. Seeley, T. D. Stephens, and P. Tate, Anatomy and Physiology, McGraw-Hill, Boston, Mass, USA, 7th edition, 2006.

[17] Z. Y. Oo, R. Deng, M. Hu et al., "The performance of primary human renal cells in hollow fiber bioreactors for bioartificial kidneys," Biomaterials, vol. 32, no. 34, pp. 8806-8815, 2011.

[18] M. Huijuan, W. Xiaoyun, Y. Xumin, W. Hengjin, and S. Xia, "Effect of continuous bioartificial kidney therapy on porcine multiple organ dysfunction syndrome with acute renal failure," ASAIO Journal, vol. 53, no. 3, pp. 329-334, 2007.

[19] Food and Drug Administration CDRH BSE Working Group, Guidance for FDA Reviewers and industry Medical Devices Containing Materials Derived from Animal Sources (Except for in vitro Diagnostic Devices), 1998, http://www.fda.gov/MedicalDevices/DeviceRegulationandGuidance/GuidanceDocuments/uc m073810.htm.

[20] S. Jockenhoevel, G. Zund, S. P. Hoerstrup et al., "Fibrin geladvantages of a new scaffold in cardiovascular tissue engineering," in Proceedings of the 14th Annual Meeting of the EuropeanAssociation-for-Cardio-Thoracic-Surgery, Frankfurt, Germany, 2000.

[21] J. F. Mano, G. A. Silva, H. S. Azevedo et al., "Natural origin biodegradable systems in tissue engineering and regenerative medicine: present status and some moving trends," Journal of the Royal Society Interface, vol. 4, no. 17, pp. 999-1030, 2007.

[22] A. Mol, M. I. Van Lieshout, C. G. Dam-De Veen et al., "Fibrin as a cell carrier in cardiovascular tissue engineering applications," Biomaterials, vol. 26, no. 16, pp. 3113-3121, 2005. 
[23] Q. Ye, G. Zünda, P. Benedikta et al., "Fibrin gel as a three dimensional matrix in cardiovascular tissue engineering," in Proceedings of the 13th Annual Meeting of the European-Association-forCardio-Thoracic-Surgery, Glasgow, Scotland, 1999.

[24] R. Büttemeyer, J. W. Mall, M. Paulitschke, A. Rademacher, and A. W. Philipp, "In a pig model ePTHE grafts will sustain for 6 weeks a confluent endothelial cell layer formed in vitro under shear stress conditions," European Journal of Vascular and Endovascular Surgery, vol. 26, no. 2, pp. 156-160, 2003.

[25] T. R. Dunkern, M. Paulitschke, R. Meyer et al., "A novel perfusion system for the endothelialisation of PTFE grafts under defined flow," European Journal of Vascular and Endovascular Surgery, vol. 18, no. 2, pp. 105-110, 1999.

[26] M. Hu, M. Kurisawa, R. Deng et al., "Cell immobilization in gelatin-hydroxyphenylpropionic acid hydrogel fibers," Biomaterials, vol. 30, no. 21, pp. 3523-3531, 2009.

[27] T. K. Ip, P. Aebischer, and P. M. Galletti, "Cellular control of membrane permeability. Implications for a bioartificial renal tubule," ASAIO Transactions, vol. 34, no. 3, pp. 351-355, 1988.

[28] Y. Duan, N. Gotoh, Q. Yan et al., "Shear-induced reorganization of renal proximal tubule cell actin cytoskeleton and apical junctional complexes," Proceedings of the National Academy of Sciences of the United States of America, vol. 105, no. 32, pp. 11418-11423, 2008.

[29] M. Essig, F. Terzi, M. Burtin, and G. Friedlander, "Mechanical strains induced by tubular flow affect the phenotype of proximal tubular cells," American Journal of Physiology, vol. 281, no. 4, pp. F751-F762, 2001.

[30] K. J. Jang and K. Y. Suh, "A multi-layer microfluidic device for efficient culture and analysis of renal tubular cells," Lab on a Chip, vol. 10, no. 1, pp. 36-42, 2010.

[31] H. Zhang, F. Tasnim, J. Y. Ying, and D. Zink, "The impact of extracellular matrix coatings on the performance of human renal cells applied in bioartificial kidneys," Biomaterials, vol. 30, no. 15, pp. 2899-2911, 2009.

[32] M. Ni, J. C. M. Teo, M. S. B. Ibrahim et al., "Characterization of membrane materials and membrane coatings for bioreactor units of bioartificial kidneys," Biomaterials, vol. 32, no. 6, pp. 1465-1476, 2011.

[33] J. C. M. Teo, R. R. G. Ng, C. P. Ng, and A. W. H. Lin, "Surface characteristics of acrylic modified polysulfone membranes improves renal proximal tubule cell adhesion and spreading," Acta Biomaterialia, vol. 7, no. 5, pp. 2060-2069, 2011.

[34] M. Wieser, G. Stadler, P. Jennings et al., "hTERT alone immortalizes epithelial cells of renal proximal tubules without changing their functional characteristics," American Journal of Physiology, vol. 295, no. 5, pp. F1365-F1375, 2008.

[35] P. M. T. Deen, S. Nielsen, R. J. M. Bindels, and C. H. Van Os, "Apical and basolateral expression of Aquaporin-1 in transfected MDCK and LLC-PK cells and functional evaluation of their transcellular osmotic water permeabilities," Pflugers Archiv European Journal of Physiology, vol. 433, no. 6, pp. 780-787, 1997. 


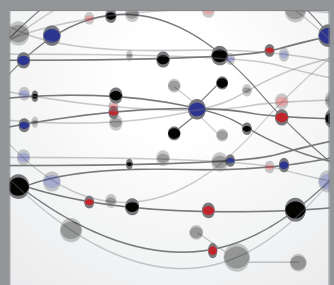

The Scientific World Journal
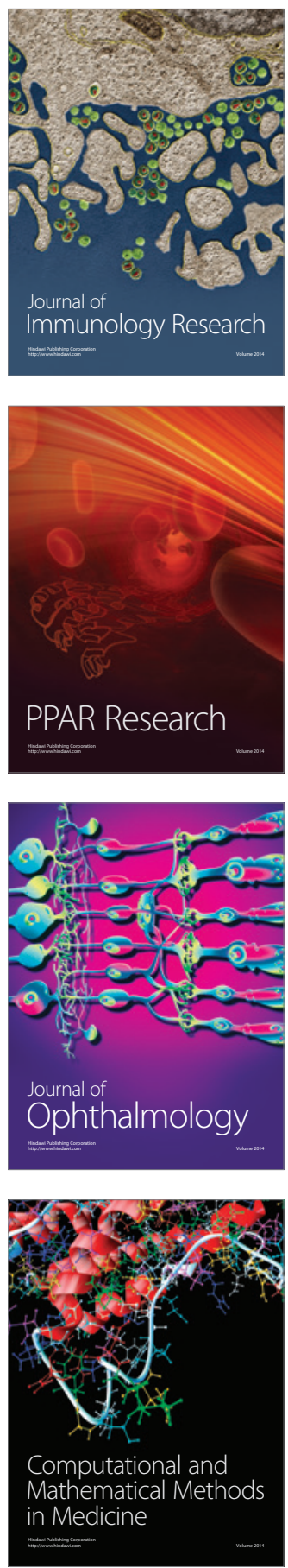

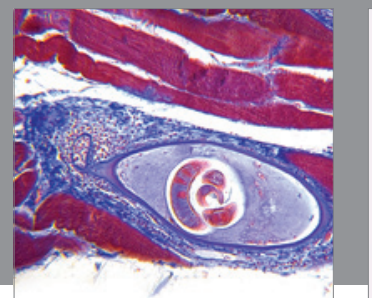

Gastroenterology

Research and Practice
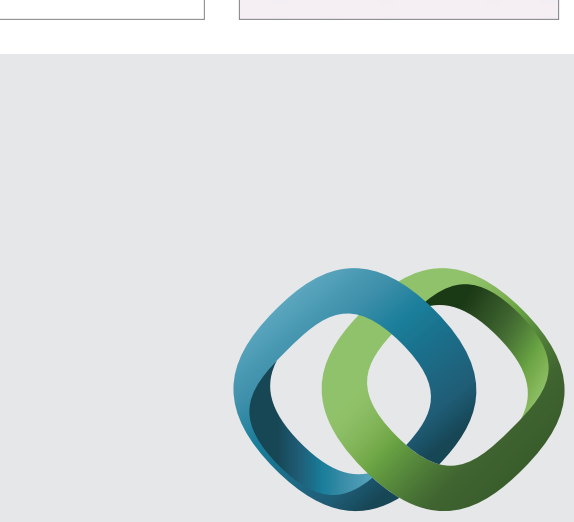

\section{Hindawi}

Submit your manuscripts at

http://www.hindawi.com
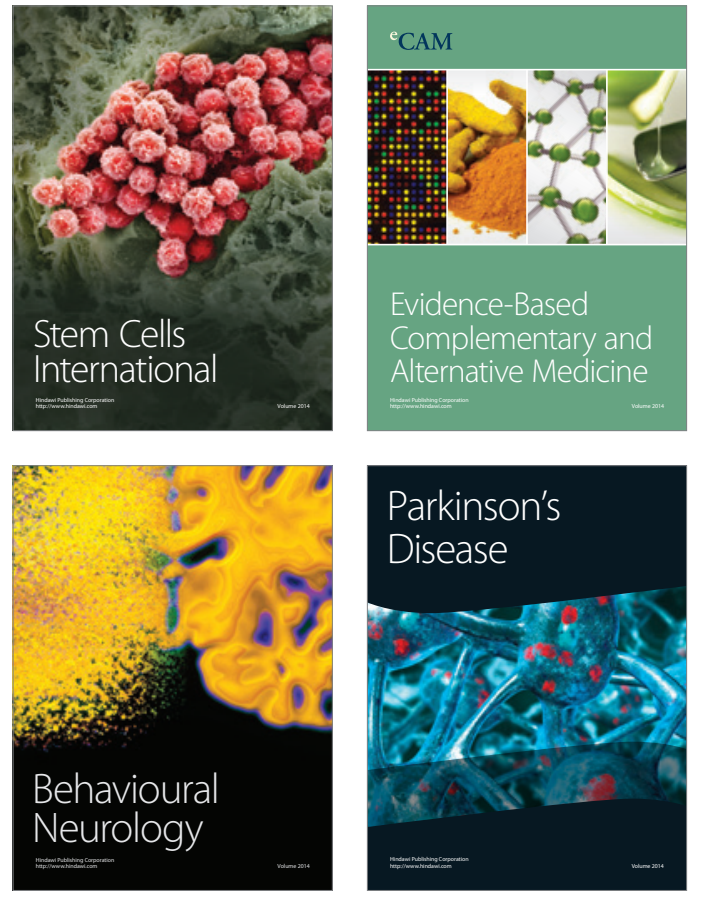
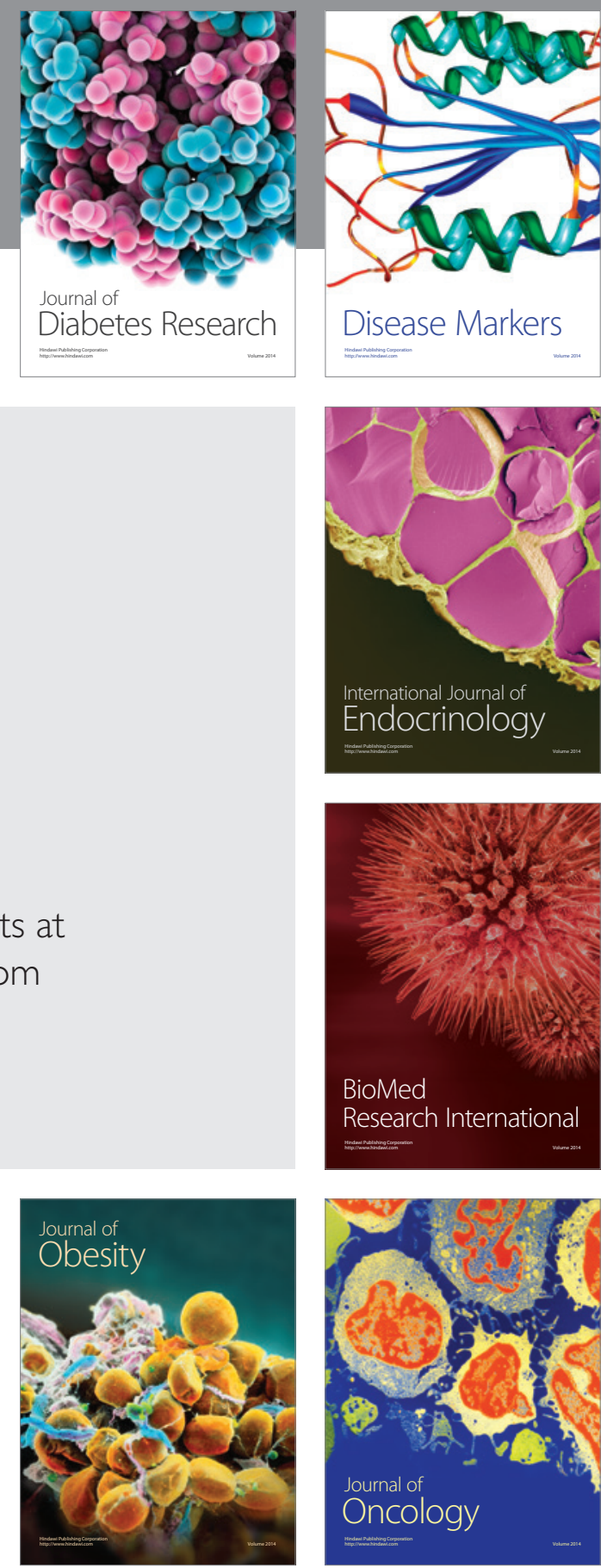

Disease Markers
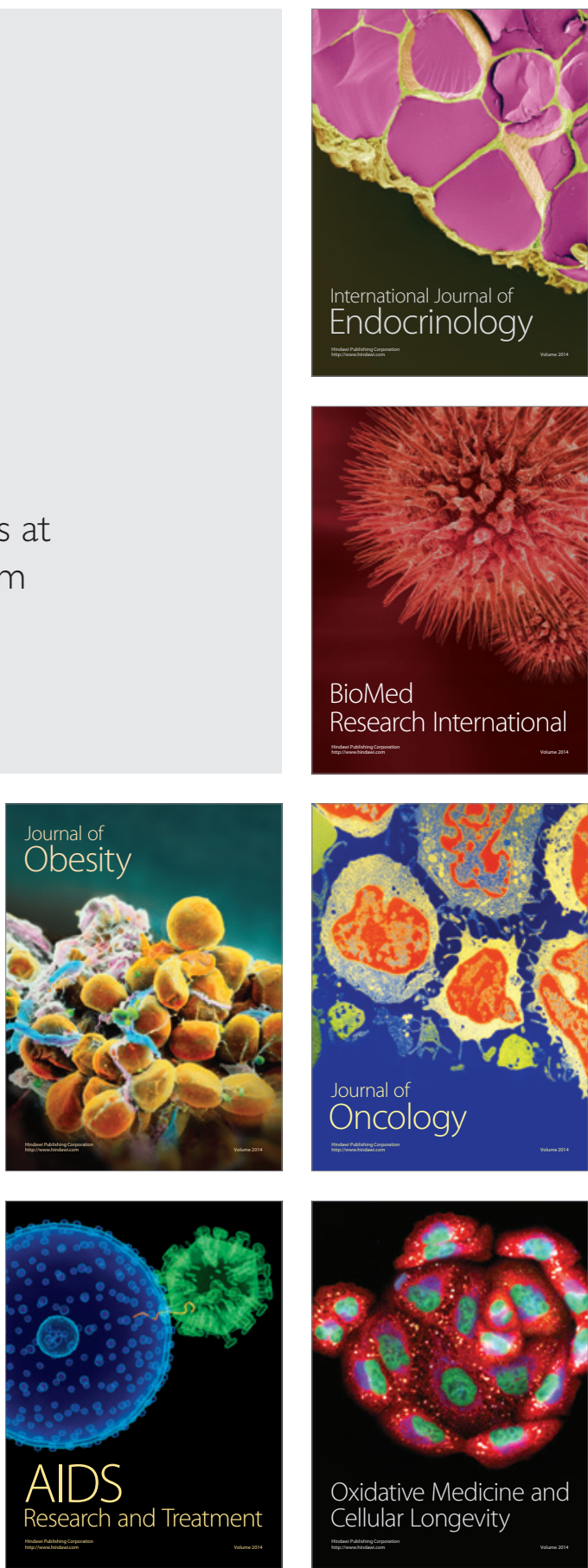